\title{
CONSENTIMIENTO Y DELITOS CONTRA LA VIDA HUMANA DESDE LA PERSPECTIVA CONSTITUCIONAL
}

\section{Consent and crimes against human life from a constitutional perspective}

Felipe Jiménez. C.

\begin{abstract}
Resumen: En este artículo se pretende analizar, críticamente, el rol que asigna la mayoría de la dogmática nacional y comparada al consentimiento de la víctima en los delitos contra la vida humana independiente. Para ello se inicia el análisis desde la perspectiva filosófica acerca del problema de la disponibilidad de la vida. Luego, desde una óptica liberal, se analiza el concepto de bien jurídico y su conexión con la Constitución y el texto constitucional. Se evalúa el rol del consentimiento dentro de la teoría del delito, apartándose de la distinción tradicional entre acuerdo y consentimiento propiamente tal. Finalmente, se plantean modificaciones a la situación actual en el derecho penal chileno a partir de la consagración constitucional de los principios de dignidad y autonomía de la persona humana.
\end{abstract}

Palabras clave: consentimiento - homicidio a petición - eutanasia - autonomía dignidad humana.

Abstract: In this paper the author tries to critically analyze the role that most of the criminal law authors in Chile and abroad concede to the victim's consent in crimes against independent human life. In order to achieve this goal, the author takes as a starting point the philosophical perspective towards the problems regarding voluntary disposition of life as a right. Later, the author analyses the concept of 'protected interest' from a liberal point of view, and its connection with the constitutional order. The role of assent is evaluated within the criminal law theory, separating from the classical distinction between assent and agreement. Finally, the author proposes modifications to the current situation in Chile's criminal law, taking as a starting point the constitutional consecration of the principles of human dignity and autonomy.

Keywords: consent - requested homicide - euthanasia - autonomy - human dignity.

\section{Introducción}

La discusión acerca del rol que pueda cumplir el consentimiento de la víctima como motivo suficiente para excluir la punibilidad es una de las más relevantes de la dogmática penal actual. En especial, cuando dicha discusión se centra en la posibilidad de disposición del bien jurídico protegido en los llamados "delitos

\footnotetext{
* Abogado, Pontificia Universidad Católica de Chile. Correo electrónico: fnjimene@gmail.com. El autor quisiera agradecer los comentarios y sugerencias de Crescente Molina, Andrés Peñaloza y Marcela Prieto a versiones anteriores de este texto, así como los valiosos comentarios de un árbitro que evaluó este artículo antes de su publicación.
} 
Jiménez - Consentimiento y delitos contra la vida humana...

contra la vida", el problema se hace muy difícil de resolver. Como es evidente, ello se debe, en gran parte, a que dicha discusión está ligada a problemáticas más profundas, de carácter ético, filosófico, político e incluso religioso.

El problema es por cierto antiguo, ${ }^{1}$ sin perjuicio de que, como señala Tomás-Valiente, "la consideración de la vida como bien supuestamente 'indisponible' (...) ha constituido durante siglos un dogma inatacable del Derecho Penal que sólo en un período relativamente reciente comienza a ser puesto en cuestión". ${ }^{2}$

Pues bien, hoy la polémica excede con creces el ámbito penal y parece estar cada vez más extendida en la sociedad, de manera que el debate se ha hecho transversal y -como nunca antes- relevante en el discurso público. Como señala Dworkin, "la discusión sobre la eutanasia ha saltado de pronto a las primeras páginas de los periódicos". ${ }^{3}$ A este fenómeno ha contribuido la reciente despenalización de la eutanasia activa en algunos países. Tal es el caso de Holanda y Bélgica, que en los años 2001 y 2002, respectivamente, autorizaron la eutanasia practicada por un facultativo, con el cumplimiento de ciertos requisitos estrictos, entre los que cabe destacar, en especial, el consentimiento del paciente. ${ }^{4}$

En nuestro ordenamiento penal, la regla general consiste en que un atentado contra la vida humana independiente será castigado mediante la aplicación del tipo de homicidio simple (art. $391 \mathrm{~N}^{\circ} 2$ del Código Penal). Esta figura puede considerarse como el tipo básico, generalmente aplicable, cuando se produce el resultado de "causar la muerte a otro". Adicionalmente, existen los tipos de homicidio calificado (art. $391 \mathrm{~N}^{\circ}$ 1), parricidio (art. 390) e infanticidio (art. $394) .^{5}$

Por su parte, la intervención de un tercero en el suicidio consumado de una persona (auxilio al suicidio) -incluso una persona competente que haya tomado tal decisión de manera reflexiva- es constitutiva de delito. En efecto, el artículo 393 del Código Penal dispone que "el que con conocimiento de causa prestare auxilio a otro para que se suicide, sufrirá la pena de presidio menor en sus grados medio a máximo, si se efectúa la muerte".

El homicidio a petición, por su parte, no se encuentra consagrado de manera expesa en nuestro ordenamiento jurídico, pues era opinión del legislador penal que

\footnotetext{
${ }^{1}$ La discusión respecto a la disponibilidad de la vida se remonta al Fedón de Platón (Platón, Phd., 61e). En tanto, según Politoff, Matus y Ramírez, se atribuye a Francis Bacon el inicio de la discusión acerca de la eutanasia. Cfr. Politoff, Sergio, et al.: Lecciones de Derecho Penal chileno, v.2, 2005, p. 37.

2 Tomás-Valiente, Carmen: La disponibilidad de la propia vida en el Derecho Penal, 1999, p. 1.

${ }^{3}$ Dworkin, Ronald: El dominio de la vida, 1993, p. 9.

${ }^{4}$ Resulta bastante interesante, en todo caso, el hecho de que en Holanda no existe un deber jurídico impuesto al facultativo de realizar la eutanasia. Cfr. Politoff et al., Lecciones (nota 1), p. 43.

${ }^{5}$ En este esquema, sigo a Politoff et al, Lecciones (nota 1), p. 23.
} 
REJ - Revista de Estudios de la Justicia No 16 - Año 2012

"si alguien ayuda a morir a otro 'hasta el punto de ejecutar él mismo la muerte', tal hecho indudablemente constituye un verdadero homicidio y debe ser castigado como tal". ${ }^{6}$ Por esta razón, el homicidio a petición es sancionado mediante la aplicación de la figura común del artículo $391 \mathrm{~N}^{\circ} 2$ (homicidio simple) o, en su caso, alguna de las figuras calificadas. Con esto, nuestro derecho penal se aparta de la regulación dada en otros países, en los que se ha consagrado expresamente el tipo del homicidio a petición, tales como Alemania ( $\$ 216 \mathrm{CP}$ alemán), Holanda (artículo 293 del CP holandés), Italia (artículo 579 CP italiano) y España (artículo 143.4 CP español).

En cuanto a la eutanasia, en Chile tanto la "eutanasia pasiva" -omitir un tratamiento extraordinario con el fin de prolongar artificialmente una vida humana- como la "eutanasia indirecta" -"aceleración de la muerte del paciente a consecuencia de la administración de fármacos" con objeto de reducir el dolorestán excluidas del ámbito punible. Esto se debería, según parte importante de la doctrina nacional, al hecho de que estas situaciones se hallan dentro del ámbito de la causal de justificación de ejercicio legítimo de un derecho, en cuanto en ella se darían cursos de acción plenamente validados por la lex artis médica.'

De todas maneras, trataré este problema utilizando la dogmática que reconstruye el derecho penal en términos de protección de bienes jurídicos. Esta estrategia permite, en efecto, vincular el tratamiento jurídico penal de las situaciones descritas con la Constitución Política y los principios que ella consagra.

Por ello, abordar la eutanasia desde la perspectiva del ejercicio legítimo de un derecho ${ }^{10}$ es errado. En efecto, y atendida la gran relevancia que otorga la deontología médica contemporánea al consentimiento del paciente, ${ }^{11}$ es posible considerar que es precisamente ahí donde debe buscarse una eventual impunidad de la eutanasia, cuestión sobre la que se ahondará más adelante. ${ }^{12}$

Pero en cuanto a la eutanasia activa, esto es, terminar deliberadamente la vida de quien desea morir con el fin de evitar grandes padecimientos o bien una vida con escasas posibilidades de normalidad y autonomía, la doctrina dominante sostiene que sí sería punible, por tratarse de actos decisivamente encaminados a terminar con una vida humana. ${ }^{13}$

\footnotetext{
${ }^{6}$ Ibid., p. 41.

${ }^{7}$ Ibid.

8 Ibid., p. 39.

${ }^{9}$ En este sentido, Ibid., pp. 36 y ss.

${ }^{10}$ Perspectiva adoptada por Matus y Etcheberry, entre otros. Cfr. Ibid., p. 39.

11 Véanse al respecto los artículos 24 y siguientes del Código de Ética del Colegio Médico de Chile, el cual, por lo demás, consagra el derecho del paciente a una muerte digna en su artículo 23.

12 Véase punto 4.a.

${ }^{13}$ Esta línea de interpretación, reafirmada por la Ley $\mathrm{N}^{\circ} 20.584$, que regula derechos y deberes de los pacientes en su atención de salud, ha sido criticada recientemente por Figueroa, Rodolfo: "Matar, dejar morir y eutanasia en el proyecto de ley sobre derechos de las personas y en la doctrina chilena", en Revista Médica de Chile, 2011; 139, pp. 655-659. La crítica central a estas consideraciones
} 
Jiménez - Consentimiento y delitos contra la vida humana...

En todo caso, para el tratamiento de este tema no basta con el análisis de la tipicidad penal. Es necesario, en primer lugar, abordar la discusión filosófica respecto al problema de la disponibilidad de la vida.

\section{La disponibilidad de la vida en la discusión filosófica}

\section{a) La vida como bien jurídico indisponible}

Dentro de las tesis contrarias a la disponibilidad jurídico-penal de la vida, es posible distinguir dos líneas de argumentación muy diferenciadas. En efecto, por un lado existen posturas que, enmarcadas dentro de un discurso -al menos prima facie- democrático y moderno, abogan por la protección de la vida aun en contra del interés del titular. Por otro lado, existen concepciones que no necesariamente se inscriben dentro de dicho discurso, sino que argumentan desde lo que Rawls calificaría como una "doctrina comprensiva", ${ }^{14}$ y que no lidian adecuadamente con el hecho del pluralismo razonable. Entre estas últimas se encuentran -por ejemplo- aquéllas que fundamentan la indisponibilidad de la vida humana en argumentos de carácter religioso. ${ }^{15}$

Lo cierto es que concepciones de este último tipo difícilmente pueden hallar cabida dentro de los principios básicos en los que debiera fundarse el derecho penal de un Estado democrático. Por ello, resulta más relevante el examen crítico de la justificación de la indisponibilidad de la vida desarrollada dentro de un discurso políticamente razonable, justificación cuyos argumentos aparecen más plausibles teóricamente y susceptibles de esgrimirse en el ámbito público de una democracia deliberativa. ${ }^{16}$ Por lo demás, se trata de posturas conscientes del hecho de que, para un Derecho Penal respetuoso de los derechos individuales, la intervención punitiva debe ser debidamente justificada, más aún en los casos en que tal intervención se da en contra de la voluntad del titular de un bien jurídico de carácter personal.

Dentro de estas líneas de argumentación, la razón para la indisponibilidad de ciertos bienes jurídicos de importancia grave, tales como la vida, se encontraría según varios autores en la protección normativa de los intereses a largo plazo del

está dada por el rechazo a la distinción moral entre matar y dejar morir y a la utilización del principio de doble efecto como criterio delimitador desde el punto de vista moral.

14 Sobre la noción de doctrina comprensiva, véase Rawls, John: El liberalismo político, 1993, Conferencia II, parágrafo 3.

${ }^{15}$ Cfr. Ugarte, José Joaquín: El Derecho de la Vida: El Derecho a la vida. Bioética y Derecho, 2006, p. 118, quien considera que la indisponibilidad de la vida se funda "en que ella es obra directa de Dios, y sólo quien es causa de una realidad tiene dominio sobre la misma". En todo caso, argumentos similares han sido comunes en la historia del pensamiento filosófico. Así, por ejemplo, Pufendorf y Locke (Cfr. Ibid., pp. 118 y ss.).

${ }^{16}$ Esto se vincula directamente a la noción, seguramente conocida, de razón pública. Al respecto, véase Rawls, El liberalismo político (nota 14), Conferencia VI. 
REJ - Revista de Estudios de la Justicia No 16 - Año 2012

individuo. Para las tesis de esta índole, se "pretende proteger al individuo de su propia irracionalidad". ${ }^{17}$

En opinión de Tomás-Valiente, quien defiende en esta materia una tesis limitadamente paternalista, en definitiva "las dificultades se plantean (...) a la hora de fijar los límites de lo que la sociedad puede asumir como decisiones racionales de morir, en las que podría colaborarse (incluso ejecutivamente) sin lesionar ningún deber ético-social como el impuesto por la solidaridad". ${ }^{18}$ En este sentido, añade la autora, "no existe excesivo problema en mostrarnos antipaternalistas y partidarios del respeto a la autonomía de la voluntad cuando se trata de decisiones que, además de ser racionales desde el punto de vista de los valores o planes de vida del sujeto, lo son también desde el punto de vista social externo (...).” Más adelante advierte que, "[s]in embargo, el empleo de un razonamiento de este tipo debería (para poner a prueba su propio antipaternalismo) conducir a reflexionar sobre si se considera o no legítimo prohibir la colaboración en otro tipo de suicidios libres no restringidos al ámbito eutanásico estricto, donde la tensión entre los principios mencionados (autonomía y beneficencia) sí deviene más clara". ${ }^{19}$

Como se ve, y en esto lleva la razón Tomás-Valiente, donde mayor plausibilidad tienen los postulados favorables a la indisponibilidad de la vida es en el ámbito de casos que no pueden ser considerados como de eutanasia pasiva o indirecta en sentido estricto, sino que -como en el homicidio a petición de una persona competente- se refieren a la disposición voluntaria, libre y premeditada de la vida, con colaboración activa y decisiva de un tercero, sin la concurrencia de circunstancias que, como en el caso de la eutanasia pasiva solicitada por el paciente terminal, hagan atendible a priori la razonabilidad externa de una "voluntad de morir". En estas situaciones, precisamente, pareciera existir una prevalencia de criterios intersubjetivos, prohibitivos de la disposición de la vida ajena, por sobre la voluntad libre e informada de quien desea morir. ${ }^{20}$

\section{b) La vida como bien jurídico disponible}

Un modelo paradigmático de las argumentaciones que han intentado fundamentar teóricamente la disponibilidad de la vida es el desarrollado por Ronald Dworkin. Intentando dar una solución genuinamente liberal al problema, este autor ha dado una interesante defensa de la admisibilidad del suicidio asistido y de la eutanasia. Esta argumentación tiene como punto central la idea de que existen ciertas decisiones de tal relevancia para la construcción de la propia identidad y del sentido de la propia vida, que no deben quedar al arbitrio estatal, sino sometidas a la decisión autónoma del propio ciudadano. Entre tales decisiones cabría, según

\footnotetext{
17 Tomás-Valiente: La disponibilidad... (nota 2), p. 109.

18 Ibid., p. 121.

19 Ibid., pp. 121-122.

${ }^{20}$ Para un análisis detallado de las justificaciones específicas de la indisponibilidad de la vida, véase Ibid., pp. 30-65.
} 
Jiménez - Consentimiento y delitos contra la vida humana...

Dworkin, la decisión de cómo y cuándo morir, pues "hacer que alguien muera en una forma que otros aprueban, pero que él cree que es una contradicción horrorosa con su propia vida, constituye una devastadora y odiosa forma de tiranía". ${ }^{21}$

Para Dworkin, en el problema de la eutanasia existen tres cuestiones de carácter moral y político fundamentales: la autonomía, los mejores intereses del paciente y el carácter sagrado de la vida. ${ }^{22}$

Respecto a los dos primeros, este autor señala que es correcto que el respeto a la autonomía individual suministra un criterio claro a favor de permitir la disposición de la propia vida, mientras que el de los mejores intereses puede aportar criterios favorables a ambas posturas. ${ }^{23}$

En tanto, tratándose del carácter sagrado de la vida (el argumento más común en contra de la permisión de la eutanasia y el suicidio asistido), Dworkin sostiene que "la convicción de que la vida humana es sagrada suministra, probablemente, la base emocional más poderosa para resistirse a la eutanasia". ${ }^{24}$

Pero en opinión de este autor, es justamente el criterio de la santidad de la vida el que puede otorgar un argumento fundamental a favor de la eutanasia. En este sentido, el hecho de que la vida sea una cuestión tan sagrada e inviolable, implicaría para Dworkin la necesidad de que las decisiones más relevantes referidas a ella sean dejadas al arbitrio de las personas, pues "las opiniones se dividen, no porque algunas personas desprecien valores que otras aprecian sino, por el contrario, porque los valores en cuestión se encuentran en el centro de la vida de cualquiera, y porque nadie puede tratarlas como suficientemente triviales como para aceptar las órdenes de otras personas acerca de lo que esos valores significan". 25

Por ello, la permisión de la eutanasia puede fácilmente vincularse con el deber moral de cada sujeto de tomar las decisiones más relevantes de su vida en conformidad a sus intereses críticos (intereses que dicen relación con la concepción de sí mismo y la construcción de la propia identidad); esto es, de un modo internamente racional.

\footnotetext{
${ }^{21}$ Dworkin: El dominio... (nota 3), p. 284.

22 Ibid., pp. 248-255.

${ }^{23}$ Si bien, como el propio Dworkin señala, existirá una diferencia sustancial según si se consideran los intereses de experiencia (aquéllos vinculados con el disfrute de ciertas actividades, sensaciones o experiencias por sí mismas, por el hecho de ser placenteras) con los intereses críticos (aquéllos que dicen relación con la autocomprensión del sujeto, la construcción de su identidad y los puntos básicos en torno a los cuales él ha querido sostener su vida e incluso explicarla). En este sentido, parece relativamente claro que seguir los intereses críticos exige el debido respeto por la autonomía del sujeto.

${ }^{24}$ Ibid., p. 255.

25 Ibid., p. 284.
} 
A continuación se tratará el aspecto penal del problema.

\section{La noción de bien jurídico}

La problemática acerca del bien jurídico es una de las más discutidas históricamente en el derecho penal, lo que de todas formas no ha impedido que dicho concepto se haya erigido como una de las principales herramientas para el análisis sistemático y crítico de la legislación penal vigente. ${ }^{26}$ En todo caso, existen también sectores importantes de la dogmática penal que niegan la utilidad de esta herramienta metodológica. ${ }^{27}$

Tratándose, pues, de un concepto problemático, es necesaria una breve aclaración sobre la concepción de bien jurídico que se toma como propia, especialmente si se considera que dicha concepción puede permitir una adecuada solución del problema planteado.

Para efectos de lo planteado en este trabajo, se asume plenamente la postura de Roxin, quien "desde la Constitución y concretamente en la idea de 'estado de derecho', trata de fundamentar el carácter prepositivo de los bienes jurídicos". ${ }^{28}$ Para conceptualizar el bien jurídico, Roxin explica que "el punto de partida correcto consiste en reconocer que la única restricción previamente dada para el legislador se encuentra en los principios de la Constitución". ${ }^{29}$

En el contexto normativo actual, por lo demás, situar el concepto de bien jurídico como anterior a la Constitución es un error, precisamente porque la Constitución tiene un carácter constitutivo de la comunidad política, y "lo que define a lo constitutivo es que la práctica constituida no puede preexistir a las condiciones de su constitución". ${ }^{30}$ Por ello también es posible hablar, en el sentido de Searle, de hechos institucionales (en oposición a hechos brutos) cuando estamos frente a las prácticas de nuestra comunidad política como tal, incluyendo, por cierto, las prácticas del sistema de persecución penal. ${ }^{31}$

Por estas razones, es posible concebir como bienes jurídicos -tal como lo hace Roxin- a las "circunstancias dadas o finalidades que son útiles para el

\footnotetext{
${ }^{26}$ Respecto al concepto de bien jurídico y su discusión, véanse Hormazábal, Hernán: Bien jurídico y Estado social y democrático de Derecho 2006, p. 167, y Roxin, Claus: “¿Es la protección de bienes jurídicos una finalidad del derecho penal?”, en Hefendehl, Ronald: La Teoría del bien jurídico: ¿fundamento de legitimación del Derecho penal o juego de abalorios dogmático?, 2007, pp. 443-458.

27 Véase, por todos, Jakobs, Günther: "El principio de culpabilidad", en Anuario de Derecho Penal, Tomo XLV, Fascículo III, 1992, pp. 51-71.

${ }^{28}$ Hormazábal: Bien jurídico... (nota 26), p. 107.

${ }^{29}$ Roxin, Claus: Derecho Penal: Parte General, 1997, p. 55.

30 Mañalich, Juan Pablo: "Pena y ciudadanía", Revista Estudios de la Justicia, N6, 2005, p. 82.

31 Este punto guarda relación con la distinción entre reglas regulativas y reglas constitutivas, planteada por Searle, John: Speech Acts, 1969, pp. 33-42.
} 
Jiménez - Consentimiento y delitos contra la vida humana...

individuo y su libre desarrollo en el marco de un sistema social global estructurado sobre la base de esa concepción de los fines o para el funcionamiento del propio sistema", ${ }^{32}$ circunstancias que no pueden ser concebidas como anteriores al "sistema global estructurado" y, como consecuencia de ello, de la Constitución, la que -precisamente- se caracteriza por estructurar dicho sistema.

Esta concepción de bien jurídico, correctamente caracterizada como liberal, puede sin embargo ser afirmada sin comprometerse con un punto de vista excluyentemente liberal (por ejemplo, en el sentido de una doctrina comprensiva, tales como las de Mill y Kant según Rawls), ${ }^{33}$ pero no puede ser afirmada sin aceptar a los principios políticos liberales como la mejor interpretación posible de nuestras instituciones políticas.

Por lo demás, como señala Hormazábal, "según Roxin, en la medida que la Ley Fundamental en su artículo 20 II.1. establece que todo poder estatal emana del pueblo, no podría ponerse en duda que la actividad estatal no puede tener otro fin que el de asegurar las condiciones de vida en común (...), lo que realiza conminando con pena, bajo determinadas condiciones, la violación de bienes jurídicos". ${ }^{34}$

En el caso chileno, es posible sostener que el principio de servicialidad del Estado a la persona humana (art. $1^{\circ} \mathrm{CPR}$ ) y el principio de subsidiariedad (art. $1^{\circ}$ $\mathrm{CPR})^{35}$ conllevan una concepción limitadora de la potestad punitiva, en cuanto ella puede única y exclusivamente dirigirse a la protección de bienes jurídicos. ${ }^{36}$ Esta es, precisamente, una manifestación concreta (cuya base se encuentra en la Constitución) del principio de fragmentariedad del derecho penal: ${ }^{37}$ el derecho penal no puede abarcar todo el ámbito de conductas ilícitas, sino que sólo "aquellas que constituyen ataques intolerables en contra de bienes jurídicos cuya subsistencia es capital para la preservación de la convivencia pacífica". ${ }^{38}$ Pues bien, estos bienes jurídicos que permiten la subsistencia de la comunidad política ${ }^{39}$ sólo

\footnotetext{
32 Roxin: Derecho penal... (nota 29), p. 56.

${ }^{33}$ Rawls: El liberalismo político (nota 14), pp. 68, 109 y 177.

${ }^{34}$ Hormazábal: Bien jurídico... (nota 26), p. 107.

35 Véase una perspectiva crítica en Aldunate, Eduardo: "La desconstitucionalización de la Constitución", en Revista de Derecho de la Pontificia Universidad Católica de Valparaíso, XXII, 2001, p. 25.

36 Sobre este punto -y su vinculación con el problema de la disponibilidad- ahondaremos más adelante.

${ }^{37}$ Véase al respecto, por todos, Cury, Enrique: Derecho Penal: Parte General, 2005, pp. 88-89.

38 Ibid., p. 88.

${ }^{39}$ En la tradición contractualista, el nacimiento de la comunidad política se explica justamente por la necesidad de convivencia pacífica. Esto aparece con claridad en el pensamiento de autores como Hobbes. Véase, al respecto, Godoy, Oscar: "Selección de textos políticos de Thomas Hobbes", en Estudios Públicos, $\mathrm{N}^{\circ} 23,1986$, pp. 7-8. Igualmente, y específicamente respecto a la importancia del temor a la muerte como fundamento de la filosofía política de Hobbes, véase Strauss, Leo: La filosofía politica de Hobbes: su fundamento y su génesis, 2006.
} 
REJ - Revista de Estudios de la Justicia No 16 - Año 2012

pueden (en un derecho "sin respaldo religioso o metafísico") ${ }^{40}$ surgir de aquello que precisamente constituye a dicha comunidad: la Constitución.

Sobre este punto se ahondará más adelante.

\section{El consentimiento}

\section{a) Sobre la naturaleza jurídica del consentimiento: ¿Exclusión de la tipicidad o causal de justificación?}

Si bien se trata de una discusión que en nuestro medio prácticamente no ha sido abordada, existe un importante desarrollo en la dogmática comparada respecto al rol del consentimiento en el ordenamiento jurídico-penal. En efecto, ya sea partiendo de la disposición legal expresa en el tipo, ${ }^{41}$ de la consagración del consentimiento como causal de justificación o bien del contexto global del ordenamiento jurídico, es posible asignar un rol relevante al consentimiento respecto a la exclusión de la punibilidad.

En concreto, la discusión que se ha dado desde Geerds ${ }^{42}$ estriba en la posibilidad de distinguir entre acuerdo (excluyente del tipo) y consentimiento (como causal de justificación). La doctrina alemana dominante sigue esta distinción, ${ }^{43}$ atribuyendo distintos requisitos y efectos tanto al acuerdo como al consentimiento. Así, en cuanto a requisitos de manifestación, capacidad y ausencia de vicios del consentimiento, entre otros, el acuerdo requeriría para su constitución un estándar sustancialmente inferior al consentimiento justificante. ${ }^{44}$ Por lo demás, como señala Angulo, el acuerdo "resulta de 'naturaleza meramente fáctica' (por contraposición al consentimiento)". 45

Sin embargo, en esta materia parece llevar la razón Roxin, para quien "el consentimiento (...) deberá considerarse de forma general como excluyente de la tipicidad porque no es posible una clara delimitación de los casos de consentimiento respecto de los de acuerdo". ${ }^{46}$

\footnotetext{
${ }^{40}$ Habermas, Jürgen: Facticidad y validez: sobre el derecho y el estado democrático de derecho en términos de teoría del discurso, 1998, p. 95.

${ }^{41}$ Como ocurre, por ejemplo, en los tipos penales que expresamente consagran como un elemento del tipo la falta de consentimiento o autorización del titular del bien jurídico (así, por ejemplo, el hurto; artículo 432 del Código Penal).

${ }^{42}$ En este sentido, Roxin: Derecho Penal... (nota 29), p. 512.

43 Incluso autores como Jakobs consideran que existen: acuerdo excluyente del tipo, consentimiento excluyente del tipo y consentimiento justificante. Derecho Penal: Parte General. Fundamentos y teoría de la imputación, 1995, pp. 522 y ss.

${ }^{44}$ Respecto a los requisitos del consentimiento, véase Casas Barquero, Enrique: El consentimiento en el derecho penal, 1987.

45 Angulo, Graciela: "El consentimiento frente a los bienes jurídicos indisponibles", en Revista latinoamericana de Derecho, No 7-8, 2007, p. 64.

${ }^{46}$ Roxin: Derecho Penal (nota 28), p. 522.
} 
Jiménez - Consentimiento y delitos contra la vida humana...

Pero, además, es necesario considerar al consentimiento como excluyente del tipo -y no como causal de justificación- por razones de orden sustantivo. En efecto, no es posible considerar al consentimiento como causal de justificación si se tiene en cuenta que la idea de la tipicidad como una categoría predominantemente descriptiva ha sido hoy prácticamente abandonada, y que "la opinión predominante en la doctrina comparada estima que los tipos son tipos de imjusto (...) que describen comportamientos probibidos en general y cuya ejecución, en consecuencia, se considera en principio jurídicamente desvaliosa". ${ }^{47}$

Por ello, no parece adecuado considerar una determinada acción como típica si ella se debe a la voluntad del sujeto pasivo, titular del bien jurídico protegido disponible. ¿No resulta acaso contraintuitivo considerar como un comportamiento prohibido en general y jurídicamente desvalioso la intervención médica que implica la pérdida de un miembro del cuerpo? En efecto, ¿podemos considerar como constitutivo de lesiones (justificadas, según parte importante de la doctrina en nuestro país, ${ }^{48}$ pero lesiones al fin) la extracción de un tumor maligno? $¿$ Es dicha conducta una conducta en principio prohibida, en cuanto está dentro del ámbito de protección de la norma que prohíbe las lesiones?

Parte significativa de la doctrina comparada, fundándose en los criterios de imputación objetiva, considera correctamente que las lesiones ocasionadas en tratamientos terapéuticos no son típicas por no tratarse de riesgos prohibidos por la norma y que se encuentran, por tanto, fuera de su ámbito de protección. Sin embargo, ¿qué ocurre si la intervención quirúrgica salvadora se realiza contra la voluntad del paciente ${ }^{29}$ ¿Debiéramos considerar, dentro de un Estado de Derecho democrático, que es admisible la intervención de un tercero en la corporeidad de un sujeto competente sin su consentimiento? En realidad, en estos casos pareciera que, al menos desde el punto de vista del consentimiento, sí se trata de conductas típicas. ${ }^{50} \mathrm{Y}$ ello se debe a que, en definitiva, el bien jurídico protegido en los tipos de lesiones, más que la mera protección del objeto material, es el dominio autónomo del individuo sobre su propio cuerpo. Como correctamente señala Rudolphi, "bien jurídico y poder de disposición sobre el bien jurídico forman no

\footnotetext{
${ }^{47}$ Cury: Derecho Penal... (nota 37), pp. 286-287.

48 Así, por ejemplo, Politoff et al., Lecciones... (nota 1), pp. 120-127.

${ }^{49}$ Casos de este tipo han sido conocidos por la jurisprudencia nacional. "En 1996 la autonomía fue privilegiada como un derecho fundamental al momento de haberse rechazado el Recurso de Protección Rol N 805-96 en la Corte de Apelaciones de Santiago. Con este recurso el Hospital San José pretendió transfundir a un paciente contra su voluntad, aludiendo a que la vida era el bien superior. El fallo estableció de manera sucinta pero profunda que: 'nadie puede ser forzado a defender su propio derecho" (Retamales, Avelino: “Autonomía del paciente: Los Testigos de Jehová y la elección de alternativas a la transfusión", en Revista chilena de obstetricia y ginecología, $\mathrm{N}^{\circ}$ 71, 2006, p. 283).

${ }^{50}$ La tipicidad, por lo demás, no podría excluirse por un supuesto "consentimiento presunto". Al respecto, véase infra. Un aspecto distinto, como se señala más abajo, es que la tipicidad se excluya recurriendo a otros criterios de interpretación de los tipos penales.
} 
sólo una unidad, sino que objeto de disposición y facultad de disposición son, en su relación mutua, de por sí, el bien jurídico protegido en el tipo". ${ }^{51}$

Este criterio permite resolver casos que no se solucionan con los criterios de disminución del riesgo o del ámbito de protección de la norma, tales como los de menoscabos a la integridad física en prácticas sexuales, en prácticas deportivas y en intervenciones cosméticas (v.gr. tatuajes), en el sentido de que simplemente no serían acciones típicas justamente debido al consentimiento del titular.

Sin embargo, lo dicho no equivale a postular la punibilidad de casos en los que se da una intervención beneficiosa en el cuerpo del afectado, sin su consentimiento (el caso por antonomasia son las transfusiones sanguíneas a personas cuya religión prohíbe estas prácticas) ${ }^{53}$ cuando ella se ve correctamente excluida por otros criterios de imputación objetiva, tales como el del ámbito de protección de la norma y el de disminución del riesgo.

Pero lo que sí es posible postular es que tales intervenciones beneficiosas no son jurídicamente imperativas respecto de la propia víctima, forzándola a admitir intervenciones en su corporeidad en contra de su voluntad por sus efectos terapéuticos, tal como ha ocurrido en nuestro país por la vía del recurso de protección. ${ }^{54}$ Esta situación es una forma de paternalismo estatal incompatible con los fundamentos propios de un Estado democrático de Derecho, el cual se funda en el respeto a la dignidad y a la autonomía de las personas.

Por último, si de acuerdo a los criterios de imputación objetiva la imprudencia de la víctima excluye desde un principio la tipicidad (cuestión que se manifiesta como una clara concreción del principio de autorresponsabilidad), ${ }^{55} \mathrm{con}$ mayor razón lo hace el consentimiento de la víctima. Sobre todo cuando se trata de tipos en los que su propia construcción legislativa y dogmática implica la ausencia de consentimiento (así, por ejemplo, el hurto, la violación, la injuria, la violación de correspondencia, etc.), es indudable que el consentimiento excluye la

\footnotetext{
51 Citado por Roxin: Derecho Penal... (nota 29), p. 518, nota 26.

52 Aquí, ciertamente, se presenta el problema de la ubicación sistemática del consentimiento como elemento negativo del tipo. En efecto, parece extraño, desde el punto de vista dogmático, que conductas que en principio serían impunes a partir del criterio del ámbito de protección de la norma, pasen a ser punibles a partir de la noción del consentimiento aplicado a posteriori. Una posible solución, empero, y que en este trabajo sólo se postula provisionalmente, podría ser considerar el consentimiento como una manifestación del criterio de atribución a la víctima, excluyendo, de esta manera, la aplicación del tipo ante hechos que han contado con el consentimiento de la víctima, pero sin extender el ámbito de punibilidad a hechos cuya punibilidad, pese a no existir consentimiento, puede reconducirse a otros criterios de imputación objetiva.

${ }^{53} \mathrm{Al}$ respecto, véase Retamales: Autonomía... (nota 49).

${ }^{44}$ Véase, por ejemplo, Revista Gaceta Juridica, N 50, pág. 76, Santiago, 1995, Revista Gaceta Jurídica, No 232, Santiago, octubre 1999, pp. 107-108, y Sentencia Corte Apelaciones de Santiago de $1^{\circ}$ de diciembre de 1995, confirmada por la Corte Suprema el 18 de enero de 1996.

55 Véase, por todos, a Cancio Meliá, Manuel, Conducta de la víctima e imputación objetiva en derecho penal, 2001, p. 446
} 
Jiménez - Consentimiento y delitos contra la vida humana...

tipicidad. Pero no sólo en estos casos, pues "con razón dice Stratenwerth que la voluntad del afectado es 'significativa no sólo para la protección jurídica', sino que pertenece también al objeto protegido", ${ }^{56}$ y esto es especialmente predicable de los tipos que protegen bienes jurídicos eminentemente personales.

De esta manera, queda bastante claro que el consentimiento es un elemento negativo del tipo, puesto que una conducta que ha sido querida por un sujeto, titular de ciertos bienes jurídicos, difícilmente puede considerarse en principio prohibida. Además, una correcta interpretación de los tipos penales que protegen bienes jurídicos estrictamente individuales (fundada, como hemos visto, en los criterios de imputación objetiva) lleva necesariamente a la conclusión de que el consentimiento excluye, desde un principio, la tipicidad objetiva. Por lo demás, como se verá, una correcta interpretación de nuestra Constitución Política ofrece argumentos plausibles a favor de considerar el consentimiento en el ámbito de la tipicidad objetiva. $^{57}$

\section{b) El consentimiento en el ordenamiento jurídico chileno}

No existe, en nuestro derecho penal, una consagración con carácter general del consentimiento como exclusión de la tipicidad penal. ${ }^{58}$ Como señala Ríos, "no

\footnotetext{
${ }^{56}$ Roxin: Derecho Penal... (nota 29), p. 518.

57 Un análisis interesante, desde la perspectiva de la teoría de las normas, se puede ver en Kindhäuser, Urs: Teoría de las normas y sistemática del delito, 2008, pp. 13-38. Kindhäuser rechaza, simultáneamente, la noción de que es posible conceptualizar al consentimiento como una causal de justificación y aquella que lo considera un elemento negativo del tipo. Para este autor, el consentimiento operaría entonces como una razón de cancelación de la norma. En este sentido, "con el consentimiento decae la razón para seguir la norma abstracta en el caso concreto. Puesto que el interés protegido constituye el fundamento de la norma jurídico-penal de comportamiento, con el consentimiento resulta cancelado el fundamento de validez de la norma en referencia al caso". Sin embargo, no parece del todo convincente la construcción de Kindhäuser; él mismo rechaza, por una parte, considerar atípico un comportamiento realizado con el consentimiento del titular del interés protegido, puesto que "una lesión corporal en el sentido del tipo respectivo, así como un daño de cosa sigue siendo, a pesar del consentimiento, un daño de cosa. Lo que decae es más bien la razón para omitir por mor del seguimiento de la norma, el actuar correspondiente". Sin embargo, precisamente cuando desaparece el fundamento justificante de la existencia de una norma de comportamiento, no sólo decae una razón necesaria para su seguimiento, sino que para su aplicación; y este decaimiento de las razones para la aplicación no se da, como en el caso de una causal de justificación, en la existencia de un interés que colisione con la prohibición, sino que en un interés cuya presencia implica que la prohibición ya no tiene razón de ser. Kindhäuser, entonces, está en lo correcto cuando señala que el consentimiento opera como una razón para la cancelación de la norma. Pero ello no implica la negación del carácter de elemento negativo del tipo del consentimiento, sino que más bien explicita la razón por la cual tiene el carácter, en general, de condición suficiente para la exclusión de la punibilidad. Por esto, además, el aporte de Kindhäuser, muy relevante desde el punto de vista teórico, no soluciona adecuadamente, a mi parecer, el problema de la ubicación sistemática, dentro de la teoría del delito, del consentimiento.

${ }^{58}$ En nada se ve modificada esta situación por la reciente entrada en vigencia de la Ley $\mathrm{N}^{\circ} 20.584$, que regula derechos y deberes de los pacientes en su atención de salud. Si bien esta ley se refiere al consentimiento informado y a la voluntad del paciente en estado de salud terminal, ella no consagra el consentimiento como criterio de exclusión de la punibilidad de los casos focales examinados en este trabajo. Por el contrario, ella sólo consagra con carácter general el derecho de toda persona a
} 
REJ - Revista de Estudios de la Justicia No 16 - Año 2012

existiendo en la legislación nacional una disposición que lo contemple, algún efecto del consentimiento del interesado 'sólo podría colegirse del conjunto de disposiciones del Código Penal y de otras normas de nuestro ordenamiento que explícita o implícitamente lo tienen considerado", ${ }^{59}$

En otros ordenamientos jurídicos, en cambio, el consentimiento sí es considerado expresamente. Así, por ejemplo, en el Código Penal Alemán ( $\$ 228$ ) se consagra expresamente que "quien efectúe una lesión personal con el consentimiento del lesionado, entonces sólo actúa antijurídicamente, cuando el hecho a pesar del consentimiento vaya en contra de las buenas costumbres." Por el contrario, si el hecho no atenta en contra de las buenas costumbres, el consentimiento excluye la antijuridicidad de las lesiones. En el caso italiano, en tanto, el consentimiento está consagrado con carácter general en el artículo 50 del Código Penal, que dispone que "no es punible quien ofende o pone en peligro un derecho, con el consentimiento de la persona que podía disponer válidamente del mismo".

Lo señalado no obsta, en todo caso, a que sea posible dar cabida al consentimiento en nuestro derecho penal, como una causa de exclusión de la tipicidad. Es necesario analizar, entonces, desde qué punto de vista es viable considerar que una eventual renuncia al derecho a la vida está permitida en nuestro ordenamiento jurídico.

En primer lugar, tal como se ha señalado más arriba, la noción de bien jurídico (previa al legislador) sólo es derivable, en un sistema sin fundamento trascendente, de lo que nos constituye normativamente como comunidad política: la Constitución.

Pues bien, nuestra Constitución ha consagrado expresamente en su artículo $1^{\circ}$ que "las personas nacen libres e iguales en dignidad y derechos", y que "el Estado está al servicio de la persona humana". Dentro de este ordenamiento constitucional, dignidad y autonomía aparecen como indisolubles: no puede ser digno quien es usado como medio para otro; no puede ser digno quien no está autorizado para conducir su vida de acuerdo a sus propios fines sin dañar a otro. ${ }^{60}$ Esta es una interpretación adecuada de la Carta Fundamental, puesto que la

denegar su voluntad, sin que dicho rechazo pueda, de acuerdo al artículo 14, "tener como objetivo la aceleración artificial de la muerte, la realización de prácticas eutanásicas o el auxilio al suicidio" (lo que no hace más que reforzar la interpretación tradicional en esta materia) y la facultad de toda persona de rechazar un tratamiento que prolongue artificialmente su vida (lo que, de acuerdo a lo expresado más arriba, una correcta interpretación de las reglas penales preexistentes permitía desde antes). Al respecto, véase una perspectiva crítica en Figueroa, op.cit. (nota 13).

${ }^{59}$ Ríos, Jaime: "El consentimiento en materia penal", en Política Criminal, No 1, 2006, p. 3.

60 Un argumento similar, en el sentido de deducir la autonomía de la dignidad personal, se encuentra en Bascuñán, Antonio: "La píldora del día después ante la jurisprudencia", en Revista Estudios Públicos, $\mathrm{N}^{\circ}$ 95, 2004, p. 72, si bien este autor se refiere en específico a la autonomía reproductiva de la mujer. 
Jiménez - Consentimiento y delitos contra la vida humana...

libertad de decisión y acción (vinculados íntimamente a la autonomía) son emanaciones concretas de la dignidad de la persona humana. Por lo demás, el fundamento de la culpabilidad ${ }^{61}$ consiste precisamente en la posibilidad de dirigir un reproche por la falta de lealtad comunicativa ${ }^{62}$ del sujeto al mandato del derecho. ¿Cómo fundamentar moral y políticamente este reproche en que consiste la atribución de culpabilidad si no es, precisamente, en la posibilidad del sujeto de comportarse libremente (su autonomía)? Parece claro que la autonomía personal tiene una base constitucional manifiesta y que es el presupuesto material sobre el que descansa, en gran parte, la posibilidad de dirigir un reproche institucionalizado al delincuente.

Se podrá formular algún reproche a esta interpretación. Por ejemplo, se podría plantear la objeción de que, en realidad, la intención del constituyente al consagrar la dignidad de la persona humana ha sido consagrar un orden sociopolítico determinado (en el sentido de Carl Schmitt, una "determinación consciente de la concreta forma de conjunto por la cual se pronuncia o decide la unidad política"), ${ }^{63}$ y que ese orden sociopolítico es contradictorio -precisamentecon la interpretación expuesta, pues para el constituyente la dignidad de la persona humana consistiría en que se trata de un ser creado a imagen y semejanza de Dios, de quien ha recibido la vida, de la cual -en consecuencia- no puede lícitamente disponer. $^{64}$ Dejando al margen los problemas que puede acarrear una interpretación originalista de la Constitución ${ }^{65}$ (con la agravante de que, en nuestro país, plantea problemas ineludibles de coherencia y legitimidad democrática) ${ }^{66}$ esta manera de interpretar la Constitución, propia de cierto integrismo, es en sí misma contraria a la idea misma de Constitución Política. Por otra parte, incluso

${ }^{61}$ También derivado de la Constitución, como correctamente ha señalado Náquira, Jaime: "Constitución Política y fundamento material del principio de culpabilidad", en Revista Chilena de Derecho, Vol. 22, N² 2, 1995, pp. 189-200.

${ }^{62}$ En este sentido, Mañalich, Pena y ciudadanía (nota 30), pp. 64-69.

${ }^{63}$ Schmitt, Carl: Teoría de la Constitución, 2006 (=1992), p. 46.

${ }^{64}$ En este sentido, por ejemplo, Cea, José Luis: Derecho Constitucional chileno, 2002, pp. 38-43. En un sentido mucho más categórico, Soto, Eduardo: "Dignidad de la persona humana, fundamento de los derechos humanos", Revista de Derecho Público, No 41, 1987. Por lo demás, este ha sido -lamentablemente- el criterio que ha sostenido tradicionalmente la jurisprudencia de nuestros tribunales. Así, por ejemplo, la Corte de Apelaciones de Santiago ha sostenido que el derecho a la vida "de ningún modo consiste en que tengamos dominio sobre nuestra vida misma, en virtud del cual pudiéramos destruirla si quisiéramos, sino en la facultad de exigir de los otros la inviolabilidad de ella" (Sentencia de la Corte de Apelaciones de Santiago, de fecha 9 de agosto de 1980).

$65 \mathrm{Al}$ respecto, véase Dworkin, Ronald: Law's Empire, 1986, pp. 114-150; y Raz, Joseph: "La intención en la interpretación”, Doxa: Cuadernos de filosofía del derecho, $\mathrm{N}^{\circ} 20,1997, \mathrm{pp} .199-234$. En nuestra dogmática constitucional el originalismo interpretativo es una aproximación usada con frecuencia y de manera acrítica. Así, por ejemplo, al analizar el problema constitucional que podría plantear la eutanasia, uno de los textos tradicionales en la materia se limita a señalar que "hubo consenso en la Comisión para estimar que no sería permisible admitirla desde el momento que se reconoce el derecho a la vida" (Nogueira, Humberto; Pfeffer, Emilio; y Verdugo, Mario: Derecho Constitucional, Santiago, Editorial Jurídica, Tomo I, p. 203).

${ }^{66}$ Véase Vallejo, Rodrigo y Pardow, Diego: "Derribando mitos sobre el Estado empresario", Revista chilena de Derecho, Vol. 35, N 1, 2008, pp. 139-140. 
REJ - Revista de Estudios de la Justicia No 16 - Año 2012

recurriendo al propio texto constitucional, si "Chile es una república democrática" (art. $4^{\circ} \mathrm{CPR}$ ), no se puede sostener teóricamente una interpretación que se opone, precisamente, a la posibilidad de la democracia deliberativa mediante argumentos de autoridad que acuden a una deidad y a la verdad en su dimensión apelativa. ${ }^{67}$

Por lo demás, como señala Roxin, "la dignidad del hombre debe asegurar la libertad de decisión autónoma del particular y por ello no constituye un instrumento adecuado para la restricción de la libertad de acción". ${ }^{68}$

En este sentido, una interpretación adecuada de nuestra Constitución necesariamente conlleva la consideración de que el consentimiento (en cuanto manifestación de la voluntad de un sujeto que, constitucionalmente, hemos de reconocer como autónomo) sí tiene un papel relevante en las instituciones del derecho penal. Y ese papel relevante consiste, precisamente, en excluir la tipicidad objetiva, puesto que en los tipos penales, al protegerse bienes jurídicos se protegen, en la terminología de Roxin, presupuestos y circunstancias "útiles para el individuo y su libre desenvolvimiento".

\footnotetext{
${ }^{67}$ Esta noción se toma de Atria, Fernando: "La verdad y lo político (I). La verdad y su dimensión constitutiva", en Persona y sociedad, Vol. 23, N 1, 2009, pp. 21-50. Como señala este autor: "la verdad desempeña una función que llamaré 'apelativa' cuando la referencia a ella desempeña o pretende desempeñar una función argumentativa, es decir, cuando el hecho de tener una proposición determinada el estatus de 'verdadera', se ofrece como un argumento (cuando ella misma, en términos de Hobbes, es invocada como juez)". La verdad, en cambio, "desempeña una función constitutiva cuando la referencia a ella opera no dentro de la práctica argumentativa sino como uno de sus supuestos, es decir, cuando ella define el sentido de la argumentación. Sólo distinguiendo estas dos formas en que la idea de verdad aparece es posible entender la relación entre la verdad y lo político". De esta manera, es posible distinguir nítidamente estrategias o líneas argumentativas que consideran la verdad como presupuesto constitutivo (en ese sentido la verdad pasa a ser el ideal regulativo que orienta la práctica política), de otras que, a partir de un supuesto conocimiento apriorístico del contenido de la verdad, no la afirman como un presupuesto o ideal que orienta la práctica, sino como el rasgo distintivo de ciertas afirmaciones cuyo carácter verdadero, precisamente, está fuera de cuestión y no puede ser discutido dentro del contexto mismo de la práctica. La reacción más recurrente a este uso de la verdad en su dimensión apelativa es comúnmente el escepticismo, el cual -por lo demás- con generalidad pretende autoafirmarse como una postura metaética o de segundo orden. En realidad, el escepticismo es una mala estrategia para enfrentar este uso apelativo de la noción de verdad, puesto que cae en el mismo uso apelativo que critica (para, por esa precisa razón, rechazar por entero la noción de verdad) y, como ha demostrado Dworkin, es una postura moral como cualquier otra, de primer orden, y en este sentido, requiere la misma justificación -moral- que las demás. Tal vez, por estas razones, el uso argumentativo de la noción de 'verdad' (rasgo característico de cierto iusnaturalismo escolástico que sobrevive actualmente) y el rechazo escéptico de esa noción estén más cerca de lo que a primera vista pareciera: ambos pretenden sostener posturas metaéticas, de segundo orden, que sirven para respaldar (en el caso del iusnaturalismo escolástico) o rechazar (en el caso del escepticismo) ciertos juicios morales, cuando en realidad, ambos reflejan posturas morales sustantivas que requieren, igualmente, de justificación discursiva, y que no están en un plano anterior (o en un juego lingüístico distinto) al lenguaje moral de primer orden. Al respecto, véase Dworkin, Ronald: Justice for hedgehogs, 2010, pp. 23-68.

${ }^{68}$ Roxin, Derecho Penal... (nota 29), p. 532.

${ }^{69}$ Ibid., p. 56.
} 
Jiménez - Consentimiento y delitos contra la vida humana...

En definitiva, entonces, no es posible considerar correcta la pretensión de proteger bienes jurídicos (que por definición se entienden, en primer término, como circunstancias útiles para el libre desarrollo del individuo) en contra del interés de una persona que, por una exigencia constitucional y sistemática, hemos de considerar libre y autónoma.

\section{Consideraciones finales}

Como se ha visto, el problema relativo al rol del consentimiento en los delitos contra la vida es de difícil solución y se halla vinculado a cuestiones profundas, que trascienden al análisis dogmático penal.

Sin embargo, se debe reiterar el hecho de que en nuestra Constitución y nuestro derecho penal una exigencia básica consiste en considerar a la persona como un sujeto digno, y en consecuencia libre y autónoma.

Por ello, lleva la razón Angulo cuando señala que "el derecho a la vida es inalienable, como así también lo son la libertad, la autodeterminación y la dignidad. Un Estado democrático de Derecho debe reconocerlos y garantizarlos. Esto implica, necesariamente, que cuando el titular de tales derechos decide de manera capaz y consciente disponer de ellos, no puede ser ignorado alegando ni 'su mejor interés', ni 'el interés de la comunidad', ni las 'buenas costumbres', ni aspectos morales o religiosos". ${ }^{70}$

Como señaló un destacado grupo de filósofos, estos casos "no requieren que la Corte (podríamos decir el Estado) haga juicios morales, éticos o religiosos sobre cómo la gente debiera aproximarse o confrontar su muerte (...). Por el contrario, ellos piden a la Corte reconocer que los individuos tienen un interés constitucionalmente protegido en hacer tales graves juicios por sí mismos, libres de la imposición de una ortodoxia filosófica o religiosa por el juez o el legislador".

Sin embargo: ¿Qué ocurre desde la perspectiva político-criminal? ¿De qué manera se debieran tratar estos casos por el legislador?

En primer lugar, es necesario reiterar que son inadmisibles las posturas que pretenden centrar la justificación de la prohibición de eutanasia y casos similares en un supuesto interés del Estado o de la comunidad, o bien en el respeto a las buenas costumbres, la moral o el "orden natural". Por lo tanto, una actitud

\footnotetext{
70 Angulo, Graciela: "El consentimiento frente a los bienes jurídicos indisponibles", Revista latinoamericana de Derecho, No 7-8, 2007, pp. 87-88.

${ }^{71}$ Dworkin, Ronald; Nagel, Thomas; Nozick, Robert; Rawls, John; Scanlon, Thomas; y Thomson, Judith Jarvis: "Brief of Ronald Dworkin, Thomas Nagel, Robert Nozick, John Rawls, Thomas Scanlon, and Judith Jarvis Thomson as Amici Curiae in Support of Respondents", Issues in Law \& Medicine, $\mathrm{N}^{\circ} 2$, 1996, p. 184. La traducción y el paréntesis son nuestros.
} 
REJ - Revista de Estudios de la Justicia No 16 - Año 2012

prohibitiva del Estado frente a la disponibilidad de la propia vida sólo puede justificarse políticamente en base a que ello sea imprescindible para la protección de bienes jurídicos, entendidos ahora no sólo como "presupuestos del libre desarrollo del individuo", sino que también como "circunstancias útiles para el funcionamiento del sistema social global estructurado". ${ }^{72}$

Por esta razón, y si bien en principio la renuncia a la protección de la vida debiera ser admisible (desde el punto de vista penal) por regla general, ello podría generar una situación tensa y problemática para el sistema jurídico-penal. En efecto, la posibilidad de que el consentimiento no se preste reflexivamente, sino que se deba a situaciones anómalas debidas a fenómenos patológicos e -incluso más- debido a la presión de personas cercanas, plantea problemas y dificultades bastante graves en esta materia. Por ello, está en lo correcto Jakobs cuando señala que "la razón de ser de la prohibición del homicidio a petición (y podríamos agregar, de la eutanasia activa) es el peligro abstracto de que la petición de muerte pueda no ser madura". ${ }^{73}$

En virtud de lo señalado, el debido respeto a la autonomía implica dos aspectos que podrían considerarse como contradictorios, pero son, en realidad, complementarios: por un lado, cuando la manifestación del consentimiento de la víctima no deja lugar a dudas respecto a su seriedad y madurez (despejando, por tanto, el peligro abstracto al que alude Jakobs), debiere excluirse la punibilidad, por una exigencia básica que deriva de los fundamentos mismos del sistema político, de la Constitución y del sistema jurídico penal. Pero, por otra parte, precisamente el respeto a la autonomía debe excluir la posibilidad de realizar juicios hipotéticos para reconstruir la voluntad de un paciente que no ha podido manifestarla. En este sentido, no parece admisible que el llamado consentimiento presunto excluya la tipicidad de hechos que, fácticamente, no han contado con la aquiescencia del titular del bien jurídico.

Finalmente, y a la luz de lo que se ha señalado hasta ahora, es relativamente claro que la situación en la legislación nacional debe ser modificada. No parece en absoluto razonable la punibilidad del auxilio al suicidio de personas competentes y maduras, precisamente porque se trata de un ámbito en el que el Estado no puede entrar lícitamente, sin pasar a llevar la autonomía individual. Por lo demás, "el principio fundamental sobre el cual se edifica la teoría de la imputación objetiva fue formulado a comienzos del siglo XIX por Hegel, al señalar que solo puede llamarse imputación - en estricto sentido- a aquello que en una conducta puede ser reconocido como mío" ${ }^{74}$ En el auxilio al suicidio, justamente lo que ocurre es que el resultado de muerte es imputable, en primer lugar, a la voluntad del suicida,

\footnotetext{
72 Roxin: Derecho Penal... (nota 29), p. 56.

73 Jakobs, Günther: Suicidio, eutanasia y derecho penal - Valencia: Tirant lo Blanch, 1999, p. 70. El paréntesis es nuestro.

${ }^{74}$ Reyes, Yesid: "Fundamentos teóricos de la imputación objetiva", en Anuario de Derecho Penal, T. XLV, Fasc. III, 1992, pp. 933-934.
} 
Jiménez - Consentimiento y delitos contra la vida humana...

siendo el que presta el auxilio simplemente un colaborador fáctico en la decisión del primero. En el homicidio a petición, ciertamente, ocurre algo similar.

En cuanto a la eutanasia activa, en tanto, el debido respeto a la autonomía del paciente exige que estas conductas se permitan. Sin embargo, ello debe darse única y exclusivamente cuando la voluntad real del paciente pueda ser conocida, y no mediante la reconstrucción hipotética (realizada por un tercero) de una supuesta voluntad del paciente.

Lo planteado se debe, ante todo, a necesidades de carácter políticocriminal, en el sentido de que el correcto funcionamiento del sistema penal (y por tanto del sistema social globalmente estructurado) puede verse seriamente afectado por las tensiones a las que estaría sometido con una tendencia generalizada a reconstruir hipotéticamente una supuesta voluntad de disposición de la propia vida. Por lo demás, el carácter de tabú de la muerte en nuestra sociedad, en cuanto a la imposibilidad de controlar su ocurrencia y circunstancias, hacen desaconsejable una permisión generalizada y abierta a la posibilidad de reconstrucciones hipotéticas. Pero esto, como se señaló más arriba, éste es un punto de vista que no alega que en principio la vida sea indisponible, sino que parte precisamente del supuesto contrario. En este sentido, en un sistema político democrático y liberal, respetuoso de la autonomía del individuo, las posturas contrarias a la disponibilidad de la vida tienen el peso de la argumentación. 
REJ - Revista de Estudios de la Justicia No 16 - Año 2012

\section{BIBLIOGRAFÍA}

* ALDUNATE, Eduardo: "La desconstitucionalización de la Constitución", en Revista de Derecho de la Pontificia Universidad Católica de V alparaíso, XXII, 2001.

* ANGUlO, Graciela: "El consentimiento frente a los bienes jurídicos indisponibles", Revista latinoamericana de Derecho $\mathrm{N}^{\circ}$ 7-8, 2007.

* ATRIA, Fernando: "La verdad y lo político (I). La verdad y su dimensión constitutiva", en Persona y sociedad, Vol. 23, $\mathrm{N}^{\circ}$ 1, 2009.

* BASCUÑ́́n, Antonio: "La píldora del día después ante la jurisprudencia", Revista Estudios Públicos, $\mathrm{N}^{\circ}$ 95, 2004.

* CANCIO MELIÁ, Manuel: Conducta de la víctima e imputación objetiva en derecho penal, JM Bosch Editor, Barcelona, 2001.

* CASAS Barquero, Enrique: El consentimiento en el derecho penal, Universidad de Córdoba, Córdoba, 1987.

* CEA, José Luis: Derecho Constitucional chileno, Ediciones Universidad Católica de Chile, Santiago, 2v., 2002.

* Cury, Enrique: Derecho Penal: Parte General, Ediciones Universidad Católica de Chile, Santiago, 2005.

* DWORKIN, Ronald: El dominio de la vida, Ariel, Barcelona, 1993. Justice for hedgehogs, Harvard University Press, Cambridge, 2010.

Law's Empire, Harvard University Press, Cambridge, 1986.

* Dworkin, Ronald; Nagel, Thomas; Nozick, Robert; Ralws, John; Scanlon, Thomas; y Thomson, Judith Jarvis: "Brief of Ronald Dworkin, Thomas Nagel, Robert Nozick, John Rawls, Thomas Scanlon, and Judith Jarvis Thomson as Amici Curiae in Support of Respondents", Issues in Law \& Medicine, № 2, 1996.

* FigueroA, Rodolfo: "Matar, dejar morir y eutanasia en el proyecto de ley sobre derechos de las personas y en la doctrina chilena", en Revista Médica de Chile, 2011.

* GODOY, Oscar: "Selección de textos políticos de Thomas Hobbes", Estudios Públicos, No. 23, 1986.

* HABERMAS, Jürgen: Facticidad y validez: sobre el derecho y el estado democrático de derecho en términos de teoría del discurso, Trotta, Madrid, 1998.

* HERrERA, Carlos: "La polémica Schmitt-Kelsen sobre el guardián de la Constitución”, Revista de Estudios Políticos, N 86, 1994.

* HORMAZÁBAL, Hernán: Bien jurídico y Estado social y democrático de Derecho, LexisNexis, Santiago, 2006.

* JAKOBS, Günther: Suicidio, eutanasia y derecho penal, Tirant Lo Blanch, Valencia, 1999. Derecho Penal: Parte General. Fundamentos y teoría de la imputación, Marcial Pons, Madrid, 1995. 1992.

"El principio de culpabilidad", Anuario de Derecho Penal, Tomo XLV, Fascículo III,

* JUANATEY, Carmen: "La disponibilidad del derecho a la vida en la jurisprudencia y en la legislación de los Estados Unidos de América”, Jueces para la democracia, Nº 45, 2002.

* KINDHÄUSER, Urs: Teoría de las normas y sistemática del delito, Ara Editores, 2008.

* MAÑAliCH, Juan Pablo: "Pena y ciudadanía”, Revista Estudios de la Justicia, N6, 2005.

* NÁQUIRA, Jaime: "Constitución Política y fundamento material del principio de culpabilidad", Revista Chilena de Derecho, Vol. 22, N 2, 1995.

* NOgueIRA, Humberto; Pfeffer, Emilio; y Verdugo, Mario: Derecho Constitucional, Santiago, Editorial Jurídica.

* RAWLS, John: El liberalismo Político, Crítica, Barcelona, 1993.

* RAZ, Joseph: "La intención en la interpretación", Doxa: Cuadernos de filosofía del derecho, $\mathrm{N}^{\circ} 20,1997$.

* Retamales, Avelino: “Autonomía del paciente: Los Testigos de Jehová y la elección de alternativas a la transfusión”, Revista chilena de obstetricia y ginecología, No 71, 2006. 
Jiménez - Consentimiento y delitos contra la vida humana...

* REYES, Yesid: "Fundamentos teóricos de la imputación objetiva", Anuario de Derecho Penal, T. XLV, Fasc. III, 1992.

* RÍOS, Jaime: "El consentimiento en materia penal", Política Criminal, No 1, 2006.

* ROXIN, Claus: “¿Es la protección de bienes jurídicos una finalidad del derecho penal?”, en Hefendehl, Ronald: La Teoría del bien jurídico: ¿fundamento de legitimación del Derecho penal o juego de abalorios dogmático?, Marcial Pons, Madrid, 2007.

"Tratamiento jurídico penal de la eutanasia", Revista electrónica de ciencia penal y criminología, disponible en http://criminet.ugr.es/recpc/recpc 01-10.html, 1999.

Derecho Penal: Parte General, Civitas, Madrid, 5v., 1997.

* POLITOFF, Sergio; Matus, Jean Pierre; y Ramírez, María Cecilia: Lecciones de Derecho Penal chileno, Santiago, Editorial Jurídica, 2005.

* SCHMITT, Carl: Teoría de la Constitución, Ed. Alianza, 2006 (=1992).

* SEARLE, John: Speech Acts, Cambridge University Press, Cambridge, 1969.

* SOTO, Eduardo: "Dignidad de la persona humana, fundamento de los derechos humanos", Revista de Derecho Público, No 41, 1987.

* STRAUSS, Leo: La filosofía politica de Hobbes: su fundamento y su génesis, Fondo de Cultura Económica, Buenos Aires, 2006.

* TOMÁS-VALIENTE, Carmen: La disponibilidad de la propia vida en el Derecho Penal, Centro de Estudios Políticos y Constitucionales, Madrid, 1999.

* UgARTE, José Joaquín: El Derecho de la Vida: El Derecho a la vida. Bioética y Derecho, Editorial Jurídica de Chile, Santiago, 2006.

* VAllejO, Rodrigo y PARDOW, Diego: "Derribando mitos sobre el Estado empresario", Revista chilena de Derecho, Vol. 35, N 1, 2008. 